express melanoma risk among cancer patients as compared with the general population.

Results 4101 patients with a first invasive cancer were subsequently diagnosed with a melanoma. In the first year after diagnosis, risk of being diagnosed with melanoma was more than 10-fold higher among skin cancer patients as compared with the general population ( $\mathrm{SIR}=16.595 \% \mathrm{CI} 15.1$ to 17.9). The risk dropped to 6.9 (SIR) (95\% CI 6.4 to 7.5 ) 2-5 years post-diagnosis. After non-skin cancer, this drop is much less significant, from SIR $=1.6$ (95\% CI 1.4 to 1.7$)$ to $S I R=1.4(95 \%$ CI 1.3 to 1.5$)$ in $0-1$ years and $2-5$ years after first cancer, respectively. No trend of SIR during the study period was observed.

Discussion and Conclusion Intensive clinical check-up among skin cancer patients may explain the inflated risk of second melanoma, especially in the first year post-diagnosis. Our findings suggest that increased surveillance and awareness were not the sole explanation for the rising incidence of melanoma in the Netherlands.

\section{P2-160 RIGHT-SIDE SHIFTING OF SECOND COLORECTAL CANCER-IMPLICATIONS FOR AETIOLOGY AND CLINICAL RELEVANCE}

doi:10.1136/jech.2011.142976i.95

${ }^{1} \mathrm{~L}$ Liu, ${ }^{* 2} \mathrm{~V}$ Lemmens, ${ }^{3}$ de Hingh, ${ }^{1} \mathrm{E}$ de Vries, ${ }^{4} \mathrm{M}$ van Leerdam, ${ }^{1,2} \mathrm{~J}$ W Coebergh, II Soerjomataram. 'Department of Public Health, Erasmus University Medical Center, Rotterdam, The Netherlands; ${ }^{2}$ Comprehensive Cancer Center South (IKZ), Eindhoven, The Netherlands; ${ }^{3}$ Department of Surgery, Catharina Hospital, Eindhoven, The Netherlands; ${ }^{4}$ Department of Gastroenterology and Hepatology, and Internal Medicine, Erasmus University Medical Center, Rotterdam, The Netherlands

Background Colorectal cancer (CRC) is a heterogeneous disease: cancers in proximal, distal colon and rectum show differences in carcinogenesis pathways (proximal colon cancer related to microsatellite instability vs distal colon cancer to allelic losses), epidemiological patterns and clinical characteristics. By assessing the risk of second primary CRC among CRC patients, we aimed to shed light on the aetiology of multiple CRCs and its clinical relevance.

Material and Methods We analysed the risk of second CRC among 123253 first CRC patients from the Netherlands Cancer Registry data. Standardised incidence ratio (SIR) was computed to compare risk of second CRC among CRC patients with the general population.

Results During a median follow-up of 2 years, 2720 second CRC were diagnosed in CRC patients. More than $50 \%$ of second CRC were located in the proximal colon translating into a fourfold $R R$ when compared with general population (SIR=4.1, 95\% CI 3.9 to 4.3). Although we did not observe specific high risk pairs between sub sites of the first and second CRC, interestingly, right-side predomination of second CRC risk was clear. The right-side shifting of second CRC persists even after 10 years of follow-up in all patients' sub-groups that is, synchronous and metachronous cancers, by follow-up time and sub-sites of first CRC.

Discussion and Conclusion Our results highlighted the crucial role of microsatellite instability in the development second CRC. Due to the persistently elevated risk of a proximal colon cancer, surveillance on this specific site is recommended, preferably using FOBT.

\section{P2-161 INCREASED MELANOMA RISK AMONG MELANOMA PATIENTS}

doi:10.1136/jech.2011.142976i.96

${ }^{1} \mathrm{~L}$ Liu, ${ }^{*} \mathrm{R}$ van der Leest, ${ }^{3} \mathrm{~T}$ Nijsten, ${ }^{1,2} \mathrm{~J}$ W Coebergh, ${ }^{1,3} \mathrm{E}$ de Vries. ${ }^{1}$ Deparment of Public Health, Erasmus University Medical Center, Rotterdam, The Netherlands;
${ }^{2}$ Comprehensive Cancer Center South (IKZ), Eindhoven, The Netherlands; ${ }^{3}$ Department of Dermatology, Erasmus University Medical Center, Rotterdam, The Netherlands

Background Study on risk of subsequent melanoma among melanoma patients is scarce.

Objective To investigate melanoma risk in melanoma patients. Material and Methods Netherlands Cancer Registry incidence data (1989-2008) were employed. Patients $(\mathrm{N}=57817)$ diagnosed melanoma in this period were followed. Follow-up censored at second melanoma, death or end of study. Standardised incidence ratio (SIR) was used expressing RR compared to the general population. Sexand follow-up-specific SIR were computed under for both first and second in situ and invasive melanomas. Cox regression model which contains age at diagnosis, sex, Breslow thickness, and follow-up periods was constructed to estimate relative excessive risk melanoma risk.

Results During a median of 3 yrs of follow-up time, 1985 patients diagnosed for a second melanoma. After both in situ and invasive melanoma, inflated risk for a second melanoma was observed: after a first in situ melanoma, the risk to develop a subsequent melanoma was 16.3 (SIR), 95\% CI 14.7 to 17.9. Following an invasive melanoma, risk was 13.3 (SIR), 95\% CI 12.6 to 14.1. Elevated risks were observed in all follow-up periods $(0-1 \mathrm{yr}, 2-5 \mathrm{yr}, 6-10 \mathrm{yrs}$, $10-15$ yrs, $16-20$ yrs) in both sexes. Age at diagnosis, sex, Breslow thickness, were shown to explain the excess risk observed in those follow-up periods.

Discussion and Conclusion Based on the persistently high risk of melanoma among melanoma patients, a long-term follow-up schedule is needed for both patients and clinicians.

\section{P2-162 BONE ACCRUAL AT THE FOREARM DURING ADOLESCENCE: IS PREVENTION LIKELY TO WORK?}

doi:10.1136/jech.2011.142976i.97

\author{
${ }^{1,2} \mathrm{R}$ Lucas, ${ }^{*}{ }^{1,2} \mathrm{M}$ Severo, ${ }^{1,2} \mathrm{E}$ Ramos, ${ }^{1,2} \mathrm{H}$ Barros. ${ }^{1}$ Department of Hygiene and \\ Epidemiology, University of Porto Medical School, Porto, Portugal; ${ }^{2}$ Institute of Public \\ Health of the University of Porto, Porto, Portugal
}

Introduction Whether bone quality is essentially modifiable or tracks predictably up to adulthood is unclear. We aimed to identify a biological timing when interventions may have optimal impact on future bone properties.

Methods Participants were 709 girls from a cohort of adolescents born in 1990 and evaluated at 13 and 17 years-old. Evaluations consisted of structured questionnaires including gynaecologic age (GA) and physical evaluations comprising height, weight, body composition (bioelectric impedance) and bone mineral density (BMD) at the forearm (dual-energy x-ray absorptiometry). Our outcome was $\mathrm{BMD}$ variation during follow-up. We used linear regression to estimate its associations with baseline $\mathrm{BMD}$ and anthropometry, in four GA classes at baseline: $\leq-1,0,1$ and $\geq 2$ years relative to menarche.

Results Mean (95\% CI) annual BMD variation from 13 to 17 yearsold was highest in the earliest GA group $\left(0.030(0.028 ; 0.032) \mathrm{g} / \mathrm{cm}^{2}\right.$ year) and lowest in the oldest GA (0.018 (0.016; 0.019)). Pearson's correlation between baseline $\mathrm{BMD}$ and its increase varied from -0.12 in the youngest to -0.45 in the oldest GA. After adjustment (baseline $\mathrm{BMD}$, weight, fat mass and height) and standardisation, $\mathrm{BMD}$ variation in the lowest GA was associated with baseline BMD (-2.70 (-5.29; -0.108) per SD), but essentially with baseline weight $(11.4(0.523 ; 22.2))$ and fat mass $(-10.0(-19.2$; $-0.912)$ ). In the highest $\mathrm{GA}, \mathrm{BMD}$ variation was strongly determined by baseline $\mathrm{BMD}(-5.44(-7.20 ;-3.69))$ but not by anthropometric variables. 